\title{
Les formations herbacées de Corse. II. Essai de validation de la typologie par la caractérisation de la structure végétale des types
}

\author{
F Volaire ${ }^{1 *}, M_{\text {Godron }}^{2}$, F Lelièvre ${ }^{3}$ \\ 1 INRA, station de recherches agronomiques, 20230 San Giuliano; \\ 2 Institut de Botanique, 163 rue Auguste Broussonnet, 34000 Montpellier ; \\ э INRA, station d'amélioration des plantes, Domaine de Melgueil, Chemin de Mézouls, 34130 Mauguio, France
}

(Reçu le 28 septembre 1989 ; accepté le 7 janvier 1990)

\begin{abstract}
Résumé - Sept types de formations herbacées de Corse définis dans un article précédent sont analysés en termes de structure végétale. Cela nous permet de proposer des éléments de validation de la typologie, d'une part sur la base de comparaisons d'analyses en composantes principales et de classifications hiérarchiques; d'autre part, grâce à une analyse factorielle discriminante. Les principaux paramètres significatifs de la structure végétale des 7 types sont présentés et il est montré que la valeur pastorale et la composition du spectre biologique quantitatif (abondance des espèces pérennes par rapport à celle des espèces annuelles) différencient bien les types. Des hypothèses d'évolution des formations soumises à différentes pressions de gestion sont discutées afin d'envisager une meilleure utilisation de ces milieux.
\end{abstract}

formation herbacée / typologie / structure végétale / valeur pastorale / Corse

Summary - The herbaceous grasslands of Corsica. II. Typology validation test by the characterization of the structure of the vegetational communities. The structure of 110 samples of herbaceous grasslands is classified as belonging to 7 types (defined in a previous article; see table 1). A comparison of various statistical analyses is made to validate this typology using 20 parameters for vegetational structure (table II). A comparison between 3 principal component analyses shows that the grouping of 110 samples into 7 types permits at least $50 \%$ of the basic information to be retained. Two cluster analyses, the first based on 110 samples, and the other on the average data for the 7 types, gave rather similar results (fig 1a and 1b). A classificatory discriminant analysis shows that by using the 20 vegetational structure parameters, $72.5 \%$ of the samples can be correctly assigned to the 7 types (table III). Analysis of variance for the 20 parameters tested shows that in $80 \%$ the hypothesis of equality of the 7 types is not valid $(P=95 \%)$. The pastoral value and composition of the biological spectrum (importance of the perennial species versus the annual species) are highly differentiated from one another. All these elements can provide an argument in favour of the validity of our typology. The main significant variables in the structure of the vegetation of the 7 types are described (table V, fig 2a and 2b). Hypotheses regarding the evolution of the grasslands as a result of different modes of management are discussed in order to determine a more optimal use of these areas.

\section{grassland / typology / vegetational structure / pastoral value / Corsica}

\section{INTRODUCTION}

Dans un article précédent, nous avons montré l'importance des formations herbacées spontanées pour l'élevage extensif corse et. présenté une typologie régionale de ces milieux, basée sur 147 relevés phyto-écologiques réalisés entre 0 et $1000 \mathrm{~m}$ d'altitude (Volaire et al, 1990). Sept grands types de formations ont été définis sur des critères mésologiques et floristiques (ta- bleau 1). Ces formations étant à vocation pastorale, il est utile d'analyser les caractères de structure végétale déterminant leur valeur agronomique. Dans le prolongement des travaux de Delpech $(1960,1962)$, nous définissons la structure végétale d'une phytocénose comme «l'ensemble des paramètres traduisant des catégories d'espèces constitutives du tapis végétal". Ces variables énumérées en tableau II, sont exprimées en nombre de taxons et en contribu-

\footnotetext{
* Correspondance et tirés à part
} 
Tableau I. Caractérisation mésologique des 7 types de formations herbacées, le groupe 8 n'étant pas assimilé à un type (Volaire et al, 1990).

\begin{tabular}{lccccccccc}
\hline Types & 1 & 2 & 3 & 4 & 5 & 6 & 7 & Groupe 8 & Total \\
\hline $\begin{array}{l}\text { Conditions hydriques } \\
\text { Artificialisation }\end{array}$ & $\begin{array}{c}\text { sèches } \\
\text { nulle } \\
\text { Intensité de pâturage }\end{array}$ & $\begin{array}{c}\text { sèches } \\
\text { nulle } \\
\text { forte }\end{array}$ & $\begin{array}{c}\text { sèches } \\
\text { feu } \\
\text { faible }\end{array}$ & $\begin{array}{c}\text { saines } \\
\text { nulle } \\
\text { forte }\end{array}$ & $\begin{array}{c}\text { saines } \\
\text { fauche } \\
\text { faible }\end{array}$ & $\begin{array}{c}\text { saines } \\
\text { fertili } \\
\text { correcte }\end{array}$ & $\begin{array}{c}\text { humides variable } \\
\text { variable }\end{array}$ & $\begin{array}{c}\text { saines } \\
\text { nulle } \\
\text { faible }\end{array}$ & \\
$\begin{array}{l}\text { Nombre de relevés } \\
\% \text { de relevés }\end{array}$ & 10 & 14 & 9 & 33 & 19 & 15 & 10 & 24 & 134 \\
\hline
\end{tabular}

Tableau II. Définition et codification des 20 variables de structure végétale ( $/ S=$ indice spécifique de qualité fourragère avec $0 \leq(S \leq 5)$.

\begin{tabular}{llll}
\hline Catégories & & Nombre & Contribution \\
\hline $\begin{array}{l}\text { Composition } \\
\text { floristique }\end{array}$ & Graminées & NGR & CGR \\
& Légumineuses & NLE & CLE \\
Spectre \\
biologique & Hémicryptophytes & NHE & CHE \\
& Thérophytes & NTH & CTH \\
Gpectre & Géophytes & NGE & CGE \\
agronomique & Graminées (IS=4-5) & NG4 & CG4 \\
& Graminées (IS=1-2-3) & NG1 & CG1 \\
& Autres esp fourragères & NAF & CAF \\
& Esp de urefus" (IS=0) & NRE & CRE
\end{tabular}

Nombre total d'espèces (NES) et Valeur pastorale (VP)

tion spécifique, celle-ci pouvant être considérée comme une expression relative de la biomasse du taxon (Daget, Poissonet, 1971). Elles relèvent de 3 modes de classifications : selon les principales familles botaniques, selon les types biologiques et selon les catégories bromatologiques. Sont également pris en compte le nombre total de taxons et la valeur pastorale de chaque station (Daget, Poissonet, op cit).

L'analyse de la structure végétale des milieux étudiés se propose de répondre à 3 objectifs : tester la validité de la typologie ; caractériser la valeur pastorale des types ; enfin, présenter des hypothèses d'évolution diachronique des formations herbacées, celles-ci étant soumises à différentes modalités de gestion zoo-anthropique.

\section{MÉTHODES}

Nous utiliserons successivement des analyses statistiques plurifactorielles et monofactorielles.

\section{Analyses plurifactorielles}

\section{Analyses en composantes principales}

Nous avons réalisé 3 analyses en composantes principales (ACP). L'ACP1 part d'une matrice de données comprenant 110 lignes (relevés regroupés en 7 types) et 20 colonnes (paramètres de structure). L'ACP2 est effectuée sur les mêmes paramètres, mais relatifs à des données moyennes par type ( 7 lignes, 20 colonnes). L'ACP3 est réalisée sur le tableau de données de l'ACP2 auquel sont adjoints les 110 relevés en individus-lignes supplémentaires (117 lignes, 20 colonnes).

\section{Classification hiérarchique}

Nous avons soumis à classification hiérarchique du moment d'ordre 2 les coordonnées des variables sur les premiers axes factoriels obtenus par l'ACP1 et I'ACP2. Pour cette classification, qui est une variante des méthodes agrégatives ascendantes, le critère permettant de décider de la fusion entre classes est basé sur l'augmentation de la dispersion intraclasse (Roux, 1985).

\section{Analyse factorielle discriminante}

Enfin, par une analyse factorielle discriminante (AFD), nous avons étudié l'affectation à nos 7 types préétablis, des 110 relevés caractérisés par les 20 variables de structure de végétation. En effet, l'AFD permet de réaliser une partition optimale des relevés, en cherchant, parmi toutes les combinaisons linéaires de variables, celles qui ont une variance externe maximale et une variance interne minimale et qui seront donc les fonctions discriminantes (Lebart et al, 1982).

\section{Analyses monofactorielles}

Nous avons testé en analyse de variance, pour chacune des variables, l'hypothèse d'égalité des moyennes des 7 types. Cela nous permet de présenter une hié- 
rarchie des paramètres sur leur efficacité à discriminer globalement les types (que traduit l'indice $F$ de chaque variable).

D'autre part, des tableaux de contingence entre la variable "type" et chacun des paramètres de structure permettent de mettre en évidence les couples de modalités les plus significativement liées.

\section{RÉSULTATS}

\section{Approche méthodologique pour un test de la typologie}

\section{Analyses en composantes principales}

Une première approche de la validité de notre typologie consiste à évaluer la perte d'information induite par le regroupement des 110 relevés en 7 types. Pour cela, nous comparons les résultats de l'ACP1 et de l'ACP2 en utilisant l'ACP3. Les 4 premiers axes de l'ACP1 permettent d'expliquer $67 \%$ de la variation totale (axe $1: 26,1 \%$; axe 2 : $20,0 \%$ ). Pour les ACP2 et ACP3, la variabilité expliquée par ces 4 premiers axes est de $94,3 \%$ (axe $1: 45,2 \%$; axe $2: 22,9 \%$ ).

Cette approche (Roux, comm pers) est basée sur l'analyse des corrélations existant entre les coordonnées des 110 relevés obtenues par l'ACP1 et celles obtenues par l'ACP3, et ceci sur chacun des couples d'axes (axes 1 de l'ACP1: $F 1$, et de l'ACP3 : F'1) ; (axes 2 de l'ACP1:F2, et de l'ACP3 : F'2). Au-delà, les axes ne contribuant que plus faiblement à l'explication de la variabilité des données, ne sont pas retenus dans cette étape. Pour le premier couple d'axes (F1-F'1), le rapport de corrélation linéaire obtenu est : $r=+0,738$; pour le second couple d'axes (F2-F'2) : $r=+0,791$. L'observation des coefficients $r^{2}$, nous permet de conclure que le passage de l'ACP1 à l'ACP2 permet de conserver $54 \%$ de l'information initiale sur l'axe 1 de l'ACP2, et $62,5 \%$ de cette information sur l'axe 2 de cette même ACP.

\section{Classifications hiérarchiques des variables}

Une seconde approche cherche à comparer les hiérarchies des variables obtenues en gardant la variabilité intratype (ACP1) et en l'occultant (ACP2). Les dessins de ces 2 hiérachies, (figures $1 \mathrm{a}$ et $1 \mathrm{~b})$ sont proches. En effet, dans les 2 cas, on peut distinguer 2 principaux groupes de variables corrélées entre elles, le second groupe se dissociant, en 2 sous-groupes de variables identiques pour chacune des 2 figures $1 \mathrm{a}$ et $1 \mathrm{~b}$. La similarité de ces 2 hiérarchies apparaît
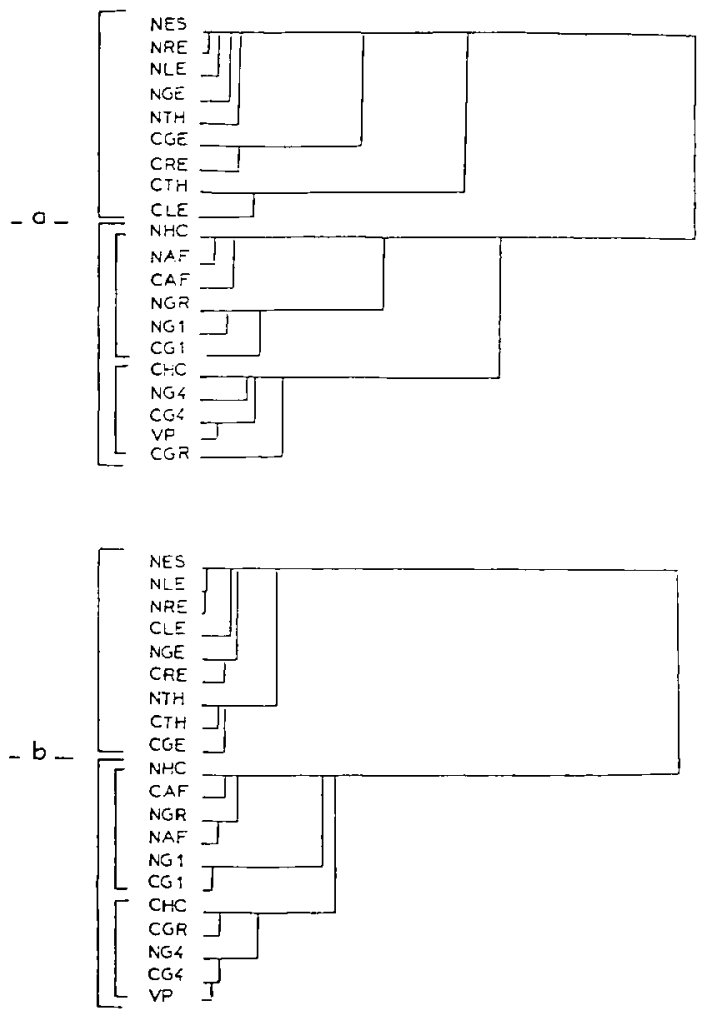

Fig 1 a et b. Dessins de la hiérarchie du moment d'ordre 2 des variables de l'ACP1 (a) et de l'ACP2 (b).

comme un élément permettant de confirmer la cohérence de la typologie réalisée.

\section{Analyse factorielle discriminante}

Le tableau III présente les pourcentages de relevés qui se retrouvent affectés au même type, d'une part selon nos propres critères de définition des types et, d'autre part, sur la base des 20 paramètres de structure végétale (AFD). On note que l'AFD permet de bien reclasser 54 à 100\% des relevés dans les types. Le chiffre le plus faible $(54,5 \%)$ est obtenu pour le type 4 qui comporte aussi le plus grand nombre de relevés ayant des caractéristiques de structure végétale intermédiaires à celles des autres types. Ce sont les relevés des milieux incendiés (type 3 ) et des formations assez humides et fertilisées (type 6) qui se regroupent le mieux en types sur la base de leı,r structure végétale (100 et $86 \%$ ). Globalement, $72,5 \%$ des relevés présentent des composantes de structure végétale qui permettent de confirmer le diagnostic typologique fondé sur des critères mésologiques et floristiques.

\section{Analyses de variance}

Comme le montre le tableau IV, $80 \%$ des variables testées permettent de rejeter l'hypothèse d'égalité des 7 types (à $p \geq 95 \%$ ) ce qui tend 
Tableau III. Classement par analyse factorielle discriminante des 110 relevés en 7 groupes, sur la base de 20 variables de structure végétale et comparaison avec la répartition des relevés selon la typologie initiale $(N R=$ nombre de relevés ; \% $=\%$ de relevés).

\begin{tabular}{|c|c|c|c|c|c|c|c|c|c|}
\hline \multirow{2}{*}{\multicolumn{2}{|c|}{ Types d'origine }} & \multicolumn{7}{|c|}{ Groupes d'affectation / AFD } & \\
\hline & & 1 & 2 & 3 & 4 & 5 & 6 & \multicolumn{2}{|l|}{7} \\
\hline 1 & $\begin{array}{l}\text { NR } \\
\%\end{array}$ & $\begin{array}{r}6 \\
60\end{array}$ & 0 & 0 & 2 & 2 & 0 & 0 & 10 \\
\hline 2 & $\begin{array}{c}\mathrm{NR} \\
\%\end{array}$ & 1 & $\begin{array}{c}8 \\
57,1\end{array}$ & 1 & 2 & 2 & 0 & 0 & 14 \\
\hline 3 & $\begin{array}{c}\mathrm{NR} \\
\%\end{array}$ & 0 & 0 & $\begin{array}{r}9 \\
100\end{array}$ & 0 & 0 & 0 & 0 & 9 \\
\hline 4 & $\begin{array}{c}\text { NR } \\
\%\end{array}$ & 5 & 3 & 1 & $\begin{array}{l}18 \\
54,5\end{array}$ & 3 & 2 & 1 & 33 \\
\hline 5 & $\begin{array}{c}\mathrm{NR} \\
\%\end{array}$ & 0 & 0 & 0 & 3 & $\begin{array}{l}15 \\
79\end{array}$ & 0 & 1 & 19 \\
\hline 6 & $\begin{array}{c}\mathrm{NR} \\
\%\end{array}$ & 0 & 0 & 0 & 1 & 0 & $\begin{array}{l}13 \\
86,7\end{array}$ & 1 & 15 \\
\hline 7 & $\begin{array}{c}\text { NR } \\
\%\end{array}$ & 1 & 0 & 0 & 0 & 2 & 0 & $\begin{array}{r}7 \\
70\end{array}$ & 10 \\
\hline Total & NR & 13 & 11 & 11 & 26 & 24 & 15 & 10 & 110 \\
\hline
\end{tabular}

Tableau IV. Classement des 20 paramètres de structure végétale par la valeur décroissante de $F$ (analyses de variance).

\begin{tabular}{lll}
\hline Paramètres & Valeur de $F \quad$ Rang
\end{tabular}

Paramètres significatifs à $p \geq 99,9 \%$ :

Contribution des Hémicryptophytes

Valeur pastorale

Nombre de Thérophytes

Contribution des Géophytes

Contribution en Graminées médiocres

Contribution en Thérophytes

Contribution en bonnes Graminées

Nombre de bonnes Graminées

Nombre d'espèces de refus

Nombre d'Hémicryptophytes

Nombre de Légumineuses

10,06
9,00
8,24
6,07
5,87
5,75
5,20
5,13
4,59

Paramètres significatifs à $p \geq 99,0 \%$ :

Nombre de Graminées médiocres

3,90

Nombre d'autres espèces fourragères

Nombre total d'espèces

3,85

Contribution des espèces de refus

$3,12 \quad 15$

Paramètres significatifs à $p \geq 95,0 \%$ :

Contribution des Graminées

Paramètres non significatifs à $p \geq 95,0 \%$ :

Nombre de Géophytes

$1,92 \quad 17$

Contribution des Légumineuses

Nombre de Graminées

0,92

Contribution d'autres esp fourragères 
également à confirmer la pertinence de la typologie. Les paramètres globalement les plus sélectifs sont les contributions spécifiques de certains types biologiques (contribution en hémicryptophytes notamment). La valeur pastorale qui se positionne au second rang de la hiérarchie apparaît comme une variable synthétique qui traduit de façon satisfaisante des différences entre types. Par contre, les catégories botaniques (nombre de graminées et contribution des légumineuses) jouent un rôle médiocre dans la discrimination globale de nos 7 types.

\section{Discussion}

Nous avons construit notre typologie sur des critères qualitatifs relatifs au milieu et à la flore. L'analyse d'un ensemble de variables quantitatives, caractérisant la structure végétale des types, a fait ressortir que :

- le regroupement des 110 relevés en 7 types conserve plus de $50 \%$ de l'information de base ;

- les distances entre variables restent très proches, que la variabilité intratype ou celle intertype soit analysée ;

- près des trois quarts des relevés peuvent être bien resitués dans leurs types d'origine par AFD.

Ces différents éléments renforcent donc la validité de la typologie.

\section{Caractérisation des types}

\section{Caractères dominants de la structure végétale}

Le tableau $\mathrm{V}$ rend compte des principales liaisons significatives entre les 7 types et les variables de structure :

- Le type 1 (milieux secs, sous-pâturés) se distingue par un faible nombre d'espèces et d'hémicryptophytes, mais la contribution en espèces de ce type biologique est significative ( 30 à $40 \%$ ), ce qui s'explique par la dominance fréquente de Dactylis glomerata.

- Le type 2 (milieux secs, surpâturés) se caractérise par une forte contribution des géophytes (15 à $40 \%$ ), en relation notamment avec le fort recouvrement de Poa bulbosa, ainsi qu'une importante participation à la biomasse des espèces de refus (30 à 40\%).

- Le type 3 (milieux secs, brûlés, sous-pâturés) se distingue par un nombre total élevé d'espèces (jusqu'à 60 taxons), avec beaucoup de therophytes, de géophytes et de légumineuses. Ceci est en accord avec les résultats de Trabaud (1981) pour les régions calcaires. Une très faible contribution des graminées de fort intérêt pasto- ral (inférieure à $5 \%$ ) apparaît également très significative de ce type.

- Le type 4 (milieux sains, surpâturés) se particularise par des caractéristiques intermédiaires à celles des autres types. Notons toutefois le fort recouvrement en espèces de refus (40 à $50 \%$ ) qui peut s'expliquer par le surpâturage auquel sont soumis ces milieux. Celui-ci entraîne le développement d'une structure végétale «en mosaïque" où les parties rases alternent avec les refus épineux proliférant sur les déjections animales (Gachon, 1979).

- Le type 5 (milieux sains, fauchés) se caractérise par de forts recouvrements en thérophytes, en graminées et notamment en graminées de faible intérêt fourrager (Vulpia myuros, Bromus sterilis). - Le type 6 (milieux sains, fertilisation azotée, gestion correcte du pâturage) se distingue par un nombre d'espèces inférieur à 30 et de fortes contributions en espèces pérennes, en particulier en graminées de bonne qualité comme Poa pratensis et Holcus lanatus.

- Le type 7 se caractérise par un faible nombre de taxons (inférieur à 30) ce qui est classique en prairies humides (Zangiacomi et al, 1978). On note également la pauvreté de la flore en thérophytes, en légumineuses et en espèces de refus, en liaison avec la nette dominance des hémicryptophytes (recouvrement supérieur à $90 \%$ ).

\section{Valeur pastorale}

Nous avons vu le rôle synthétique de la variable valeur pastorale (VP) ; la figure 2a montre que la VP moyenne est inférieure à 30 pour les formations incendiées (type 3) et les formations fauchées, peu artificialisées (type 5). La VP est comprise entre 30 et 40 pour les milieux soumis à une forte sécheresse, qu'ils soient surpâturés (type 2) ou sous-pâturés (type 1) et pour les prairies humides (type 7). Seules les stations non soumises à des stress hydriques marqués et recevant des apports minéraux (type 6) ou organiques (type 4), présentent des VP moyennes supérieures à 40 . La valeur pastorale moyenne des prairies relativement bien gérées ne dépasse pas la moitié de l'échelle des valeurs pastorales (0-10C). Cela laisse supposer un potentiel fourrager important, encore non valorisé, d'autant que le nombre moyen d'espèces de ces milieux $(35,1$; fig $2 b)$ est plus élevé que celui observé dans les prairies exploitées avec une certaine intensité dans d'autres régions. Cela indique également, que le niveau d'artificialisation pratiqué, même s'il est le plus haut rencontré pour la Corse, est encore inférieur, du fait en particulier du très faible niveau de fertilisation, à celui pratiqué dans d'autres régions herbagères. 
Tableau V. Principales caractéristiques de structure végétale des 7 types de formations herbacées : seules sont indiquées la ou les classes de descripteurs (indiquées par les valeurs représentatives de leurs bornes) qui, dans les tableaux de contingence type/descripteur, obtiennent des résultats significatifs à $p=95 \%(+$ et -$)$, à $p \geq 99 \%(++$ et $--), p=99 \%(+++$ et ---$)$ (pour la codification des variables : cf tableau II).

\begin{tabular}{|c|c|c|c|c|c|c|c|}
\hline \multirow[t]{2}{*}{ Variables } & \multicolumn{7}{|c|}{ Types } \\
\hline & 1 & 2 & 3 & 4 & 5 & 6 & 7 \\
\hline $\begin{array}{l}{ }^{*} \text { NES } \\
(0-60)\end{array}$ & ] $0-20]+$ & & $\begin{array}{l}\text { ]30-40] - } \\
\text { j40-60] + }\end{array}$ & $\begin{array}{r}\text { ]0-20] - } \\
] 30-40]+\end{array}$ & ]30-40] & $\begin{array}{l}\text { ]20-30] + } \\
] 30-40]-\end{array}$ & ]0-20) + \\
\hline $\begin{array}{l}\text { NTH } \\
(0-30)\end{array}$ & & & ]15-20] - & $\frac{] 0-5]-}{115-20]+}$ & ]15-20] + & $\underset{\text { ]15-20] }}{\text { ]0-5] }++}$ & $\begin{array}{c}\text { ]0-10] ++ } \\
\text { ]15-20] - }\end{array}$ \\
\hline $\begin{array}{l}\mathrm{NHC} \\
(0-35)\end{array}$ & ]0-10] + & $\begin{array}{r}\text { ]0-10] + } \\
\text { ]15-20] - }\end{array}$ & ]15-20] + & ] $10-15]+$ & & $\begin{array}{c}\text { j0-10] - } \\
\text { ]25-35] ++ }\end{array}$ & \\
\hline $\begin{array}{l}\text { CTH } \\
(0-90)\end{array}$ & & ]20-30] + & & $\begin{array}{c}\text { ]0-10] - } \\
\text { ]40-50] + }\end{array}$ & $\begin{array}{c}\text { J0-10] - } \\
] 60-70]+\end{array}$ & $\begin{array}{c}\text { j0-10] +++ } \\
\text { ]70-100] - }\end{array}$ & \\
\hline $\begin{array}{l}\text { CGE } \\
(0-40)\end{array}$ & $\begin{array}{c}\text { ]0-5] - } \\
{[10-15]+}\end{array}$ & $\begin{array}{c}\text { ]0-5] }-- \\
] 15-40]+++\end{array}$ & & & & $\begin{array}{l}\text { [0-5]+++ } \\
] 5-10]-\end{array}$ & \\
\hline $\begin{array}{l}\mathrm{CHC} \\
(0-100)\end{array}$ & ]30-40] + & ]10-30] ++ & & $\begin{array}{l}\text { ]50-60] + } \\
j 90-100]-\end{array}$ & ]40-50] + & ] $80-100]++$ & ]90-100] ++ \\
\hline $\begin{array}{l}\text { NLE } \\
(0-14)\end{array}$ & & ]6-8] + & $\begin{array}{l}\text { [2-4] - } \\
\text { ]8-10] ++ }\end{array}$ & & $\begin{array}{l}\text { j4-8] + } \\
\text { j8-10]- }\end{array}$ & $\begin{array}{l}\text { ]2-4] + } \\
] 6-8]-\end{array}$ & ]2-4] + \\
\hline $\begin{array}{l}\text { CGR } \\
(0-50)\end{array}$ & & & ]0-20] + & & ] $40-50]+$ & & \\
\hline $\begin{array}{l}\text { NRE } \\
(0-40)\end{array}$ & & & $\begin{array}{l}{[10-20]-} \\
] 30-40]++\end{array}$ & $\begin{array}{c}\text { ]0-10] -- } \\
] 20-30]+++\end{array}$ & ]30-40] - & $\begin{array}{l}\text { ]10-20] + } \\
\text { ]20-30] - }\end{array}$ & $\begin{array}{c}\text { j0-10] + } \\
] 20-30]--\end{array}$ \\
\hline $\begin{array}{l}\text { NG1 } \\
(0-10)\end{array}$ & & & {$[0-2]++$} & & $\begin{array}{l}\text { ]2-6]- } \\
] 6-10]+\end{array}$ & ] $2-4]+$ & \\
\hline $\begin{array}{l}\text { NG4 } \\
(0-4)\end{array}$ & & ]1-2] +++ & & $\stackrel{[0]-}{] 2-3]+}$ & $\begin{array}{l}\text { ]0-1] }+ \\
] 1-2]-\end{array}$ & $\begin{array}{l}\text { j0-1] }-- \\
j 4-5]++\end{array}$ & {$[0]+$} \\
\hline $\begin{array}{l}\text { NAF } \\
(0-6)\end{array}$ & {$[0-1]++$} & $\begin{array}{l}{[0-1]+++} \\
] 1-3]--\end{array}$ & & $\begin{array}{l}{[0-1]--} \\
] 1-2]+++\end{array}$ & $\begin{array}{l}{[0-1]---} \\
j 2-3]+++\end{array}$ & {$[0-1]---$} & ]2-3] -- \\
\hline $\begin{array}{l}\text { CRE } \\
(0-80)\end{array}$ & ]20-30]++ & ] $30-40]+$ & & $\begin{array}{l}\text { ]20-30] - } \\
] 40-50]+\end{array}$ & & {$[0-10]++$} & \\
\hline $\begin{array}{l}\text { CG1 } \\
(0-90)\end{array}$ & & ]30-40] - & & ]50-90] - & $\begin{array}{l}{[0-20]--} \\
\text { j30-90] + }\end{array}$ & & ]10-20] - \\
\hline $\begin{array}{l}\text { CG4 } \\
(0-90)\end{array}$ & & & {$[0-5]+$} & {$[0-5]-$} & $\begin{array}{c}{[0-5]++} \\
110-20]-\end{array}$ & $\begin{array}{c}{[0-5]--} \\
] 20-40]+\end{array}$ & \\
\hline
\end{tabular}

* Gamme de variation de la variable.

\section{Hypothèses sur l'évolution des formations herbacées}

Il existe peu d'études pluriannuelles de l'évolution des couverts prairiaux soumis à différentes pressions de gestion comme celle de Delpech (1975). Vu la durée et la complexité des dispositifs de suivi "diachronique", de nombreux travaux se sont orientés plutôt vers des approches «synchroniques", qui tendent à reconstituer l'évolu- tion des formations herbacées par le biais d'un échantillonnage de formations soumises à des niveaux successifs d'un facteur de milieu particulier, tel que par exemple, l'intensité d'artificialisation (de Foucault, 1980, 1986 ; Vertes, 1983 ; Balent, 1987). C'est dans cette dernière optique que nous allons présenter des hypothèses d'évolution prévisible lorsqu'une formation herbacée en conditions hydriques saines évolue : du groupe 8 (aucune gestion), au type 4 (par surpâtura- 


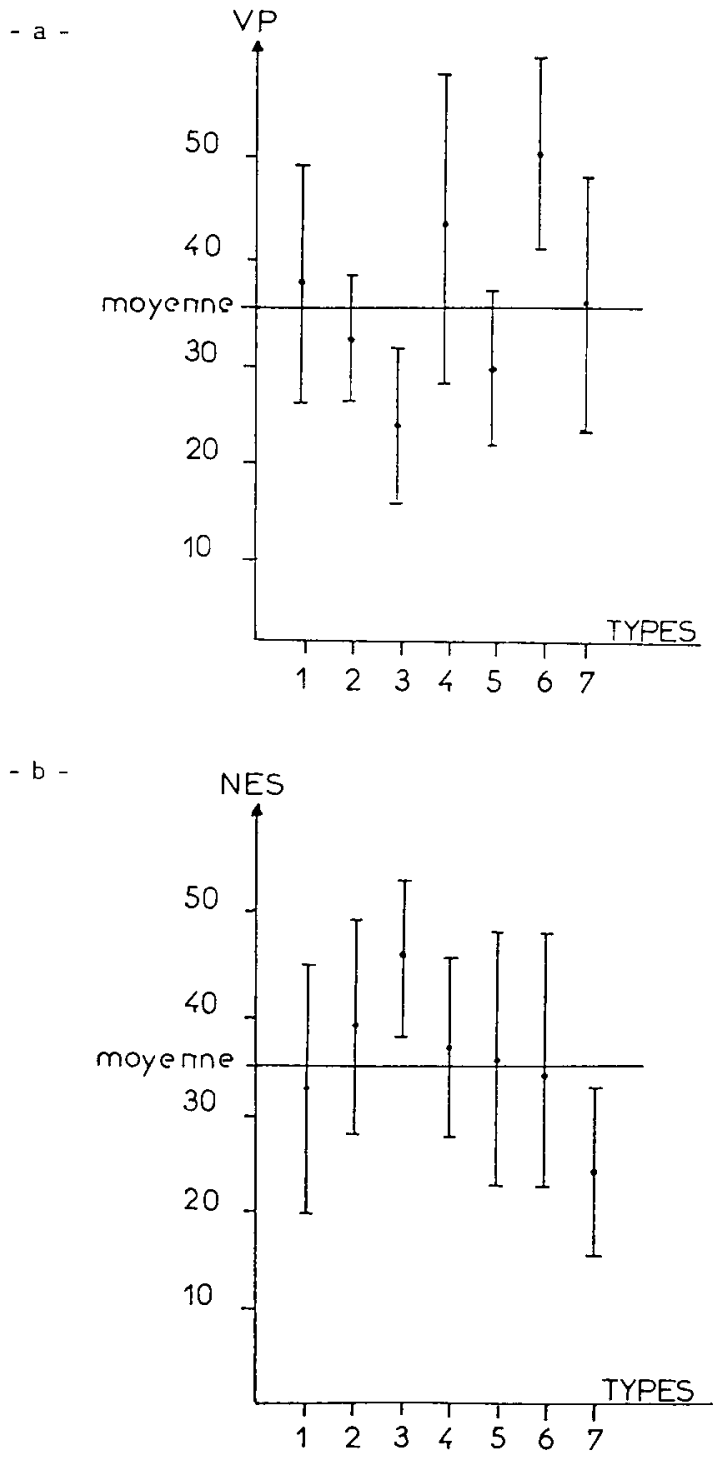

Fig 2 a et b. Moyennes et écarts types, pour les 7 types de formations herbacées, des variables :

- a. "valeur pastorale" (VP)

-b. "nombre total d'espèces" (NES).

ge), au type 5 (par fauche) et de chacun de ces cas, vers le type 6 (par fertilisation et gestion raisonnée du pâturage).

Les figures $3 a$ et $3 b$ suggèrent les hypothèses suivantes:

- La composition moyenne du spectre biologique des formations herbacées évolue de façon très significative avec le degré d'artificialisation (fig 3a). Ainsi, lorsque des friches (groupe 8) sont soumises au surpâturage (type 4), la contribution en Hémicryptophytes croît de $9,2 \%$; lorsque intervient une fertilisation azotée, le recouvrement en espèces pérennes augmente de $30 \%$ entre le type 4 et le type 6 , soit d'environ $40 \%$ en moyenne par rapport aux friches non gérées (groupe 8) ; le passage par la fauche (type 5) se traduit par une augmentation des espèces annuelles dont le recouvrement atteint $60 \%$ du recouvrement total, alors qu'en régime de surpâ-
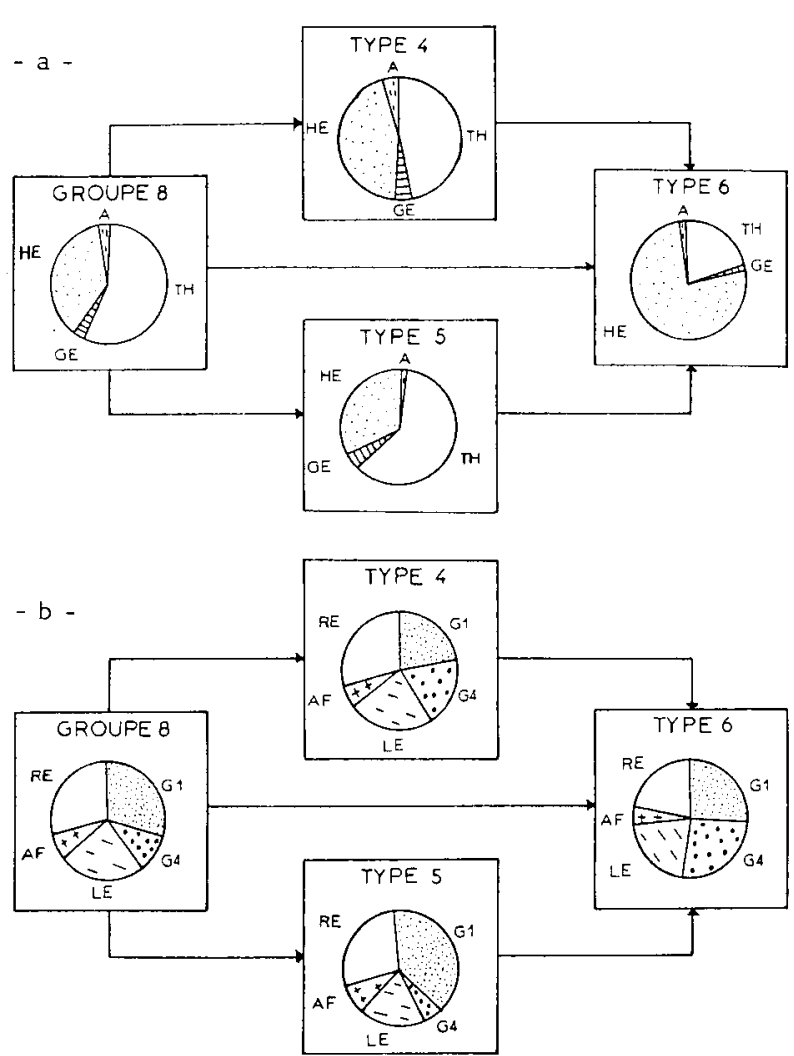

Fig 3 a et b. Tendances évolutives entre le groupe 8 et les types 4, 5 et 6 de:

- a. la contribution moyenne des espèces appartenant aux 4 types biologiques principaux ( $\mathrm{HE}=$ Hémicryptophytes, $\mathrm{TH}=$ Thérophytes, $\mathrm{GE}=$ Géophytes, $\mathrm{A}=$ Autres)

- b. la contribution moyenne d'espèces appartenant aux diverses catégories bromatologiques $(\mathrm{G})=$ Graminées médiocres, G4 = bonnes Graminées, LE = Légumineuses, $A F=$ autres fourragères, $R E=$ espèces de refus).

turage, la contribution moyenne des Thérophytes n'est que de $45 \%$.

- Les variations relatives aux catégories fourragères (fig $3 b$ ) montrent que le passage du groupe 8 (non gestion) au type 6 (fertilisation) en passant par le type 4 (surpâturage) s'accompagne de l'augmentation du recouvrement des Graminées de bonne valeur fourragère $(+16 \%$ en moyenne). A l'inverse, la pratique de la fauche, souvent tardive en Corse, stimule la prolifération des Graminées médiocres ( $11 \%$ de plus pour ces stations que pour celles du groupe 8), ceci aux dépens des Graminées intéressantes, dont le recouvrement est le plus bas des 4 groupes étudiés $(5 \%)$.

\section{Discussion}

On sait que le stade phénologique auquel la végétation est couplée, est l'un des facteurs qui peut déterminer la composition botanique de la prairie (Oomes, Mooi, 1981). La fauche tardive, telle qu'elle est pratiquée en Corse, évite le développement d'espèces ligneuses, donc l'embrous- 
saillement, mais apparaît comme un facteur de dégradation de la flore puisqu'elle stimule les Thérophytes et les Graminées médiocres, et favorise l'évolution vers des couverts prairiaux pauvres de très faible valeur pastorale (VP $=28$ en moyenne). Dans ces conditions, le passage de ces formations à des prairies bien gérées et fertilisées, peut entraîner une augmentation de qualité pastorale, mais vraisemblablement lente et progressive du fait de la faible représentativité initiale des espèces pérennes intéressantes. Par contre, la situation topographique de ces milieux est un atout : souvent situés sur des parcelles planes, mécanisables et clôturées, ils pourront être soumis, d'une façon raisonnablement envisageable, à une gestion plus intensive.

Une forte pression de pâturage apparaît relativement favorable à l'expression de bonnes valeurs pastorales ( 43,1 en moyenne). Ceci s'explique par l'abondance des déjections animales restituées sur ces milieux de faible superficie, où les animaux se concentrent. Ces apports organiques stimulent des Graminées de fort potentiel comme Lolium perenne. Toutefois ces formations ne permettent pas aux éleveurs de constituer des stocks de fourrage et leur pérennité est fonction de la charge animale variable qu'ils subissent. Celle-ci peut devenir, si elle augmente trop, un facteur de dégradation par piétinement excessif et accroissement des composées refusées, ce qu'on constate effectivement dans certaines situations du type 4. Martinand (1985) souligne en effet, que les formations pâturées stables sont rares en moyennes montagnes méditerranéennes. Dans les cas où les parcelles représentatives de ce type 4 peuvent être clôturées, les exploitants auraient intérêt à mieux utiliser ce potentiel spécifique par une fertilisation raisonnée et un patûrage tournant. Les réponses de la végétation seraient probablement rapides et intéressantes.

En ce qui concerne le groupe 8 des formations non gérées, hétérogènes et de faible valeur pastorale, des améliorations de gestion seront d'autant plus efficaces que leur fonds pastoral est plus riche ; les itinéraires techniques à rechercher semblent assez bien représentés dans le cas de la Corse, par les stations du type 6. Pour ces dernières, il est connu, qu'entre prairies permanentes soignées et prairies temporaires de plus ou moins longue durée, il existe un continuum technique (Mansat, 1983). La gestion de ces milieux relève donc de modèles d'exploitation de l'herbe (niveaux de fertilisation, rythmes de fauche et de pâture), bien connus dans d'autres régions.

II est clair que cette approche schématique ne décrit que des tendances générales qui deman- deraient à être vérifiées sur des cas expérimentaux. Ceci est d'autant plus vrai que les données moyennes relatives au groupe 8 ont été prises comme point de comparaison alors qu'elles n'ont qu'une faible valeur indicative à cause de l'hétérogénéité de ce groupe de relevés.

\section{CONCLUSION}

Après avoir défini une typologie sur des variables facilement appréciables par l'observateur sur le terrain, nous avons cherché à la tester sur des critères analytiques quantitatifs. Ceux-ci apparaissent concluants puisque, par cette approche, le classement de près des trois quarts des relevés confirme notre typologie initiale.

La caractérisation de la structure végétale des types nous conduit à envisager des schémas d'évolution possible entre les milieux et, à cet égard, il faut souligner le rôle relativement favorable du pâturage par rapport à des pratiques de fauche tardive. Par contre, le faible nombre de prairies bien gérées n'a pas permis d'affiner l'analyse de l'influence de niveaux contrastés de fertilisation ou de gestion rationnelle du pâturage sur la flore prairiale. Il s'agit là d'une des limites de cette approche descriptive. En effet, notre typologie nous paraît utilisable par les praticiens pour diagnostiquer le type auquel se rattache une formation et en estimer divers paramètres relatifs à sa valeur pastorale. Par contre, pour améliorer les pronostics que l'on peut formuler quant à l'évolution des formations après diverses interventions techniques elle doit être complétée par des références expérimentales.

\section{RÉFÉRENCES}

Balent G (1987) Structure, fonctionnement et évolution d'un système pastoral. Le pâturage vu comme un facteur écologique piloté dans les Pyrénées centrales. Thèse de doctorat ès sciences. Univ Rennes, $146 \mathrm{p}$

Daget P, Poissonet J (1971) Une méthode d'analyse phytologique des prairies. Ann Agron 22, 5-41

de Foucault B (1980) Les prairies du Bocage virois (Basse Normandie, France). Typologie phytosociologique et essai de reconstitution des séries évolutives herbagères. Doc phytosociol 5, 1-109

de Foucault B (1986) Contribution à une étude systémique des prairies de l'Aubrac (Massif central français). Doc phytosociol 11, Camerino, 255-305

Delpech R (1960) Critères de jugement de la valeur agronomique des prairies. Fourrages 4, 83-96

Delpech $R$ (1962) Possibilité de déterminer les conditions du milieu par l'examen de la végétation (cas des prairies). BTI 172, 735-749 
Delpech R (1975) Contribution à l'étude expérimentale de la dynamique de la végétation prairiale. Thèse Fac Sciences Orsay Paris, $115 p+$ annexes

Gachon L (1979) Possibilités d'évolution de l'écosystème prairial pâturé. In : Utilisation par les ruminants des pâturages d'altitude et parcours méditerranéens. $X^{e}$ journée du grenier de Theix. INRA, pp 455-459

Lebart L, Morineau A, Fenelon JP (1982) Traitement des données statistiques - méthodes et programmes. Dunod, Paris, $510 \mathrm{p}$

Mansat $P$ (1983) Les problèmes de la production d'herbe et des produits fourragers. CR Acad Agric 69 (13), 977-991

Martinand $P$ (1985) Pratiques pastorales et stratégies d'exploitation dans les moyennes montagnes méditerranéennes. BT/331-337, 399-401

Oomes MJM, Mooi $H$ (1981) The effect of cutting and fertilizing on the floristic composition and production of an Arrhenatherian elatioris grassland. Vegetatio $47,233-239$
Roux M (1985) Algorithmes de classification. Masson, Paris, $151 \mathrm{p}$

Trabaud $L$ (1981) Man and fire : impacts on mediterranean vegetation. In: Mediterranean type shrubland, (Di Castri, ed), pp 523-536

Vertes F (1983) Contribution à l'étude phytosociologique et écologique des prairies et alpages de moyenne Tarentaise. Thèse Docteur Ingénieur INA$P G, 167 p+$ annexes

Volaire F, Godron M, Lelièvre F (1990) Etude typologique des formations herbacées de Corse. I-Elaboration d'une typologie par la caractérisation mésologique et floristique des types. Agronomie 10, 163-174

Zangiacomi L, Bonischot R, Guckert A (1978) Influence de la variation du régime hydrique sur la flore et la productivité des prairies permanentes de la vallée de I'Yron (Meuse). Bull de l'ENSAIA de Nancy. Tome XX, fasc 1-2, 47-54 